\title{
CARTOGRAFÍA DE SUSCEPTIBILIDAD Y ESTIMA- CIÓN DEL MÁXIMO ALCANCE DE ALUDES EN EL CIRCO DE GREDOS (SISTEMA CENTRAL IBÉRICO)
}

\author{
Rodrigo L. SOTERES GARCÍA (rlsoteres@,uc.cl) \\ Instituto de Geografía, Pontificia Universidad Católica de Chile; Departa- \\ mento de Geodinámica, Universidad Complutense de Madrid \\ Javier PEDRAZA GILSANZ (javierp@geo.ucm.es) \\ Departamento de Geodinámica, Universidad Complutense de Madrid \\ Rosa M. CARRASCO GONZÁLEZ (carrasco@amb-to.uclm.es) \\ Departamento de Ingeniería Geológica y Minera, Universidad de Castilla- \\ La Mancha
}

Recibido: 30/11/2015

Aceptado: 20/04/2016

RESUMEN: El Circo de Gredos, Sierra de Gredos (Sistema Central Ibérico), pese a tratarse de uno de los escenarios para la práctica de deportes invernales de montaña más populares de la zona centro peninsular, no cuenta con ningún estudio dirigido al análisis del riesgo de aludes de nieve en el área. El objetivo del presente trabajo es presentar los primeros mapas de susceptibilidad de aludes de nieve y modelización del fenómeno mediante el análisis estadístico de los parámetros topográficos de recorridos de aludes conocidos en el Circo de Gredos. La cartografía confeccionada muestra que el Circo de Gredos es un área con una susceptibilidad muy alta al desencadenamiento de aludes de nieve del tipo placa y fusión.

Los cálculos realizados tras la aplicación del modelo $\alpha-\beta\left(\alpha^{\prime}=0,94 \beta+0,46 ; \mathrm{R} 2=0,94 ;\right.$ S.D. $=0,645$ ) arrojan resultados con un error máximo del $3,58 \%$ en perfiles parabólicos y del $0,99 \%$ para perfiles de transición abrupta entre las laderas y el fondo del circo, lo que invita a su utilización provisional en vista de mejorar la precisión del análisis estadístico.

Los resultados del presente trabajo muestran que el Circo de Gredos es un área con una elevada susceptibilidad de ocurrencia de aludes de nieve en la mayor parte de su superficie, situación que sumada a la intensa exposición social durante los meses invernales, generan un elevado riesgo potencial para los usuarios.

PALABRAS CLAVE: aludes, cartografía susceptibilidad, modelo, SIG, Sierra de Gredos.

\section{SUSCEPTIBILITY MAPPING AND ESTIMATION OF THE MAXIMUM RUN-OUT OF THE SNOW AVALANCHES IN THE CIRCO DE GREDOS (IBERIAN CENTRAL SYSTEM)}

ABSTRACT: The Gredos cirque, Sierra de Gredos (Central Iberian System), despite being one of the most popular mountaineering spots of the peninsular central zone does not have any assesment of the snow avalanche hazard. 
The aim of this paper is to present a collection of snow avalanche susceptibility maps and a first approach to estimate the maximum run-out distance by the statistical analysis of topographic parameters of well known avalanche paths in the Circo de Gredos. Our maps show that the Gredos cirque is an area with very high susceptibility to the triggering of avalanches, mostly slab and wet snow avalanches, due to its geomorphological characteristics and snowpack conditions.

Calculations performed after application of the model $\left(0.94 \beta \alpha^{\prime}=+0.46 ; \mathrm{R} 2=0.94, \mathrm{SD}=\right.$ 0.645 ) yield results with a maximum error of $3.58 \%$ in parabolic profiles and $0.99 \%$ for profiles with abrupt transition between the slopes and the bottom of the valley, which invites provisional use in view of improving the accuracy of statistical analysis.

The results of this work show that the Gredos Cirque is an area with a high susceptibility to occurrence of snow avalanches in most of its surface, a situation that added to the intense social exposure during the winter months generate a high risk to potential backcountry users.

KEY WORDS: snow avalanches, susceptibility mapping, numerical model, GIS, Sierra de Gredos.

\section{INTRODUCCIÓN}

Las áreas de montaña constituyen uno de los entornos naturales con una mayor susceptibilidad a experimentar diversos fenómenos de peligrosidad, entre los que destacan los aludes o avalanchas de nieve.

Aunque en España los aludes no representan un gran riesgo para la población, en las últimas décadas se ha experimentado un intenso aumento de la exposición social a estos fenómenos como consecuencia de la popularización de los deportes de invierno y los proyectos urbanísticos asociados a los mismos. Esto ha supuesto un incremento significativo del número de víctimas debido a la acción directa de los aludes (RODÉS i MUÑOZ, 1999, 2012).

En España la mayor parte de la investigación dirigida al estudio de la dinámica de aludes se ha centrado en los Pirineos (MUÑOZ TRIGO, 1988; SAÉZ ALAGÓN, 1993, 1995; FURDADA, 1996; FuRdADA y VILAPLANA, 1998; JuLIÁN y CHUECA, 1999; JULIÁN et al., 2000; CHUECA y JULIÁN, 2004; Palomo, 2007; Palomo, 2008; Chueca y Julián, 2010; Chueca et al., 2014). Fuera de los Pirineos, el número de trabajos científicos dirigidos al estudio de las avalanchas de nieve se reduce de manera considerable, siendo posible encontrar artículos dedicados a la Cordillera Cantábrica (Santos GONZÁLEZ et al., 2010) y Picos de Europa (VADA et al., 2012). Finalmente, debido a que este fenómeno no representa un riesgo generalizado en el Sistema Central, esta cadena montañosa ha tardado en sumarse al estudio de la nieve y las avalanchas (FERNÁNDEZ CAÑADAS, 2014). No obstante, su cercanía a grandes núcleos de población como Madrid, justifica la necesidad de estudios de riesgo en el área. En este contexto, la Sierra de Gredos, y en concreto el Circo de Gredos (CdG), constituye uno de los entornos con un mayor riesgo de aludes del Sistema Central, no tanto por la propia magnitud del fenómeno como por su frecuencia espacio-temporal y la gran afluencia de deportistas y 
visitantes que se acercan anualmente a practicar deportes de montaña y otras actividades de ocio.

El objetivo del presente trabajo es el estudio y análisis de los factores desencadenantes de aludes en el CdG (Ávila), tanto los vinculados con las condiciones nivometeorológicas como los relacionados con las características geomorfológicas, así como a la modelización del fenómeno en el área de estudio. El resultado final ha sido la confección de la cartografía de susceptibilidad de avalanchas y la obtención de un modelo numérico basado en parámetros topográficos adecuado para estimar el máximo alcance de los aludes en el Circo de Gredos.

\section{II. ÁREA DE ESTUDIO}

El CdG se localiza en la Sierra de Gredos, la cual forma parte del Sistema Central Ibérico (FIGURA 1) extendiéndose en dirección ENE-OSO, a través de las provincias de Ávila, Salamanca y Cáceres, funcionando como divisoria entre las cuencas del Duero y el Tajo.

Figura 1. Localización geográfica del Circo de Gredos

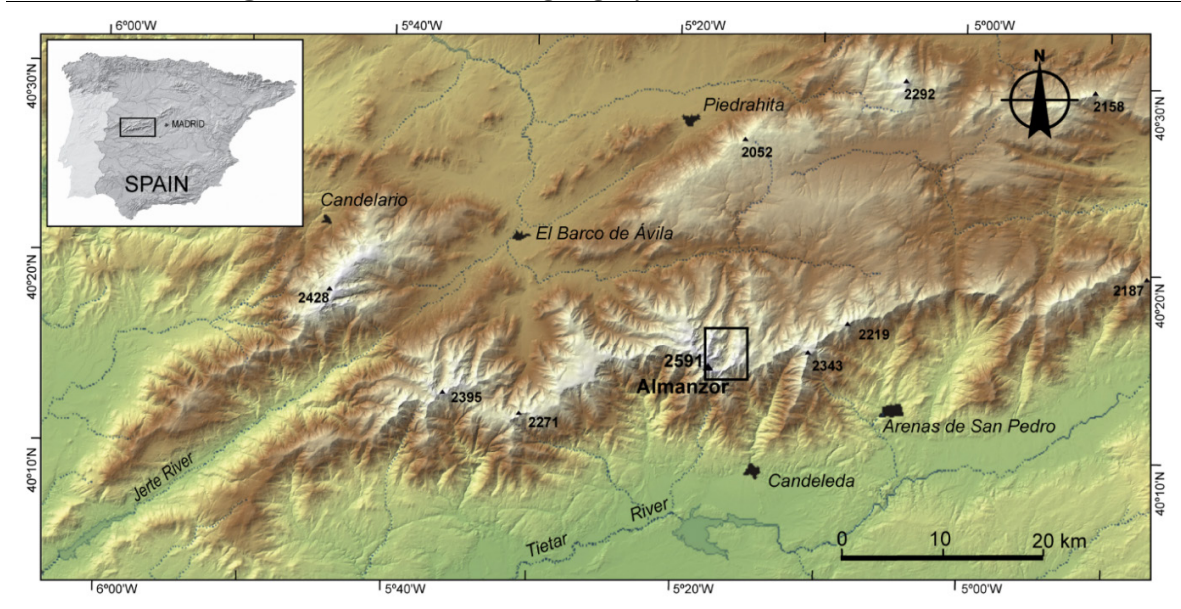

FUENTE: Elaboración propia

La litología predominante se corresponde con rocas graníticas (monzogranitos y granodioritas) con una importante abundancia de megacristales feldespáticos, además de pequeños afloramientos de rocas metamórficas migmatíticas muy evolucionadas (ODRIOZOLA et al., 1978).

El CdG constituye el mejor ejemplo de morfogénesis glaciar del Sistema Central Ibérico. Su rasgo principal es la morfología de circo, abierto hacia el 
norte, donde se encuentra la cubeta de sobre-excavación glaciar ocupada por la Laguna de Gredos, rodeada de vertientes verticalizadas, coronadas por aristas y horns, formas heredadas de las glaciaciones Pleistocenas (PALACIOS et al.,2011, 2012; CARRASCO et al., 2013, PEDRAZA et al., 2013). Las vertientes presentan pendientes muy acusadas, apareciendo frecuentemente superficies pulidas con estrías glaciares. El fondo del circo está configurado por una gran superficie de rocas aborregadas y pulidos glaciares, así como por la alternancia de campos de bloques y superficies planas cubiertas de pastos de altura, cuyo sustrato se corresponde con arenas y limos. Abundan derrubios de origen periglaciar (ACASO et al., 1985) tapizando las laderas y corredores estructurales, dando origen a numerosos conos de derrubios. La incisión observada en distintos sitios del CdG sugiere una intensa actividad morfogenética actual controlada por procesos de carácter nival y torrencial (MUÑOZ et al., 1995).

La Sierra de Gredos presenta un clima mediterráneo continental de montaña. En el caso concreto del CdG, al encontrarse situado por encima de los $2000 \mathrm{~m}$ s.n.m., la temperatura media anual oscila entre $\operatorname{los} 10$ y $2,5^{\circ} \mathrm{C}$, siendo la media de las mínimas de $-5^{\circ} \mathrm{C}$ y la media de las máximas de $27,5^{\circ} \mathrm{C}$, durante el periodo 1971-2000 (AEMET\& IMP, 2011).

Las precipitaciones medias anuales oscilan entre los 1000 y los $1400 \mathrm{~mm}$, existiendo una importante sequía durante los meses de verano (AEMET\& IMP, 2011). Cabe destacar la existencia de un significativo efecto de sombra pluviométrica entre las vertientes meridional, mucho más húmeda, y la septentrional (SARDINERO, 2004).

El número de días en los que la temperatura mínima es inferior o igual a $0^{\circ} \mathrm{C}$ sobrepasa los 100-120 días (AEMET\& IMP, 2011), situación que facilita que la cobertera nivosa alcance espesores métricos y se mantenga en el CdG, cubriendo una superficie significativa del mismo, más de 180 días al año (MUÑOZ et al., 1995), existiendo ciertos emplazamientos topográficos favorables donde los neveros pueden mantener una presencia plurianual. Las zonas de mayor acumulación de nieve se corresponden a las vertientes orientadas hacia el este y los corredores de las laderas norte y noreste.

La vegetación que recubre las laderas de la Sierra de Gredos sigue una sucesión altitudinal típica de la montaña mediterránea de clima continental. No obstante, el CdG se caracteriza por la total inexistencia de formaciones vegetales de porte arbóreo, destacando algunos parajes donde aparece un denso piornal de Cytisus oromediterraneus y las praderas de pastos alpinos de Festuca indigesta. 


\section{METODOLOGÍA}

La metodología utilizada se divide en varias etapas que incluyen: 1) observación directa en terreno; 2) el estudio de las características nivometeorológicas del CdG (SANZ et al., 2013); 3) recopilación de información histórica a través de encuestas y entrevistas personales; 4) fotointerpretación y análisis de datos espaciales digitales (FURDADA, 1996; JULIÁN et al., 2000); y 5) análisis estadístico de los parámetros topográficos incluidos en el modelo de estimación de máximo alcance del alud (LIED y BAKKEHØI, 1980; BAKKEHØI et al., 1983).

El trabajo de campo se centró en el reconocimiento de formas menores del relieve, en el estudio de los depósitos superficiales que cubren el fondo del circo y sus laderas y en el estudio de las condiciones nivometeorológicas. Este último es de gran importancia para determinar las características del manto nivoso, su evolución estacional, constatar zonas afectadas por aludes recurrentes y el funcionamiento de los corredores susceptibles de experimentarlos, así como las distancias medias que alcanzan los mismos y las características de los depósitos asociados.

La fase de recopilación de información histórica y las entrevistas personales son fundamentales para analizar la localización y frecuencia espacio-temporal y magnitud de los aludes. La primera fase consiste en un exhaustivo repaso de las hemerotecas y demás publicaciones relacionadas con el ámbito científico y montañero, donde se encontraron noticias referidas a aludes en el área de estudio en 1979. La fase de entrevistas y encuestas únicamente se ha podido realizar durante un corto periodo de tiempo (temporada 2013-2014), limitando su utilidad como base de datos. Sin embargo, los resultados son sumamente indicativos de la actividad de los aludes en esta área.

El estudio de la peligrosidad vinculado a aludes requiere de la evaluación de los factores extrínsecos (indirectos) e intrínsecos (directos) de las vertientes (MCCLUNG y SCHAERER, 1996). Los primeros hacen referencia a las condiciones nivometeorológicas, mientras que los segundos, están relacionados con los parámetros topográficos de las laderas de la zona de estudio, por lo cual son susceptibles de ser cartografiados gracias a la fotointerpretación y trabajo de campo.

La última etapa corresponde al análisis estadístico de los parámetros topográficos propuestos por LIED y BAKKEHØI (1980) y BAKKEHØIET al. (1983), los cuales están relacionados con el máximo alcance observado de los aludes y pueden ser extraídos de la cartografía. 


\section{III.1. Estudio de las características nivometeorológicas}

Se han realizado cuatro perfiles estratigráficos y de resistencia (FIGURA 2) mediante de la utilización de una sonda RAM o penetrómetro.

Figura 2. Puntos verdes: Localización de las columnas estratigráficas y perfiles de resistencia RAM. Flechas rojas: Localización de los aludes históricos y observados más frecuentemente en el $C d G$.

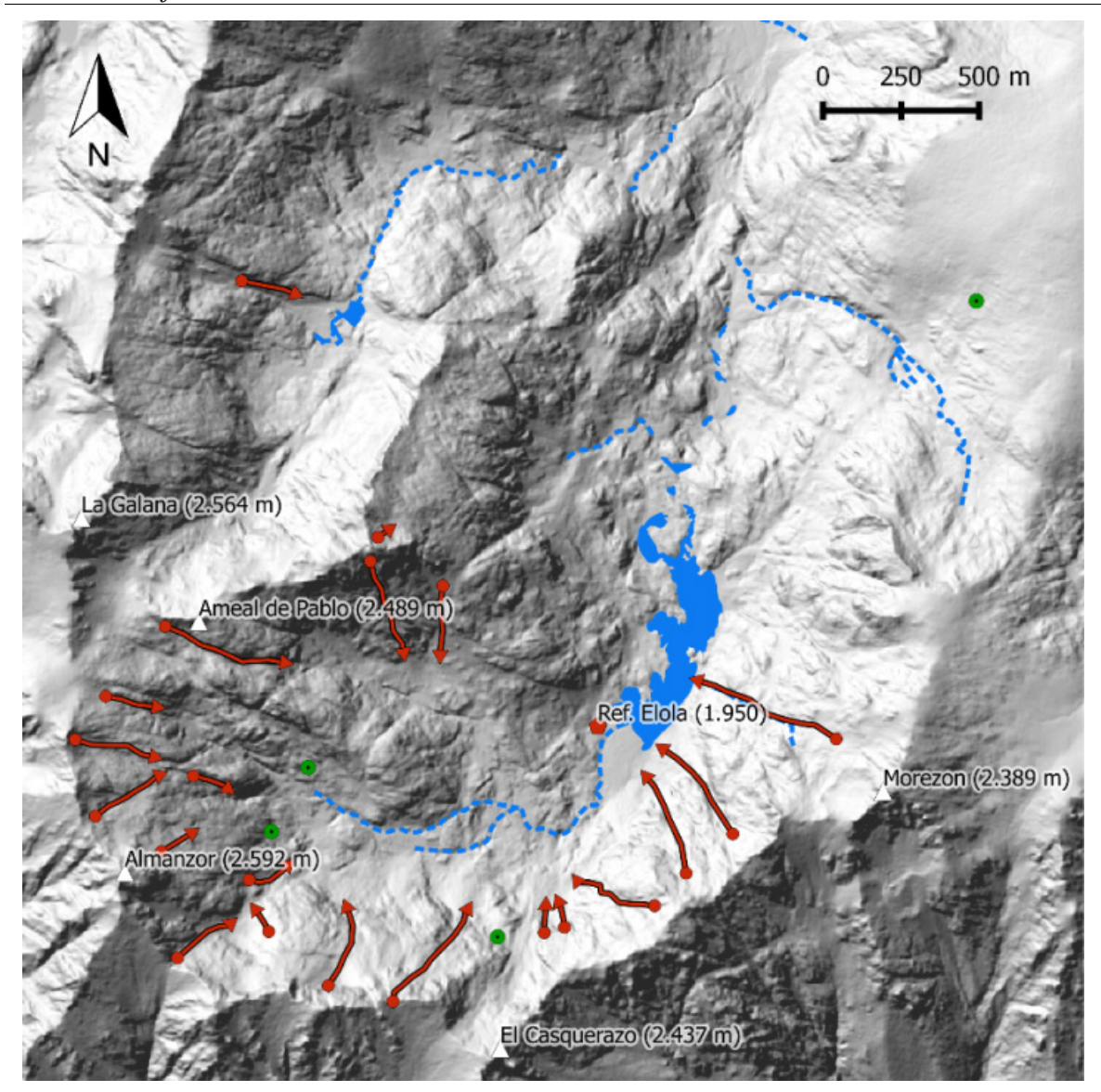

FUENTE: Elaboración propia

La selección de los emplazamientos donde realizar los perfiles y sondeos, se ha centrado en la búsqueda de áreas con características similares a las zonas frecuentes de salida de aludes (Sanz et al., 2013). Para ello se ha tenido en cuenta el espesor del manto nivoso, la altitud, la pendiente, la orientación y la lejanía de lugares de tránsito frecuente de personas que pudieran alterar las 
características del manto nivoso. De esta manera, los emplazamientos seleccionados se han situado todos por encima de los 2100 m.s.n.m, con pendientes superiores a los $30^{\circ}$, en todas las orientaciones y con al menos 1,5 metros de espesor. Los datos recogidos fueron: 1) temperatura $\left({ }^{\circ} \mathrm{C}\right)$; 2) tipo y diámetro de los granos de nieve; 3) densidad $\left(\mathrm{kg} / \mathrm{m}^{3}\right)$; 4) dureza y 5) humedad.

Las mediciones se llevaron a cabo durante el final de la temporada invernal, lo que supone encontrar un manto nivoso muy evolucionado y de gran espesor. Durante la campaña de sondeos se realizó un perfil estratigráfico y una medición de la resistencia a la penetración por orientación, lo que debe tenerse en cuenta a la hora de evaluar la influencia del manto nivoso en el desencadenamiento de los distintos tipos de aludes.

\section{III.2. Cartografía de susceptibilidad de aludes en el Circo de Gre- dos}

Para la elaboración de la cartografía de zonas probables de aludes se ha seguido la metodología empleada por Palomo et al.(2008) y Fernández Cañadas (2014), en la que los factores condicionantes para el desencadenamiento de un alud se clasifican como necesarios e intensificadores (TABLA 1).

Tabla 1. Relación de factores necesarios e intensificadores para cada tipo de alud.

\begin{tabular}{ccl}
\hline Tipo & Factores necesarios & \multicolumn{1}{c}{ Factores intensificadores } \\
\hline Placa & Pendiente $>30^{\circ}$ & $\begin{array}{l}\text { Insolación baja } \\
\text { Orientación a sotavento } \\
\text { Fusión }\end{array}$ \\
Pendiente $>25^{\circ}$ & $\begin{array}{l}\text { Permanencia de la nieve } \\
\text { Insolación alta }\end{array}$ \\
Nieve reciente & Pendiente $>45^{\circ}$ & $\begin{array}{l}\text { Permanencia de la nieve } \\
\text { Insolación baja }\end{array}$ \\
\hline
\end{tabular}

FUENTE: Adaptado de FERNÁNDEZ CAÑADAS, 2014.

Los factores necesarios son aquellos cuya ausencia disminuye la probabilidad de aludes por completo. En este caso se han considerado como tales las pendientes, desestimando la vegetación, pues en el CdG no existen formaciones vegetales con una entidad suficiente para ejercer de barrera o anclaje del manto nivoso, y la morfología (concavidad o convexidad) de las vertientes, ya que no parece mejorar los resultados cartográficos de manera significativa, pues las mayores concavidades se encuentran en áreas de $\mathrm{CdG}$ con pendientes inferiores a las necesarias para desencadenar avalanchas.

En este trabajo se considera que los aludes de nieve reciente necesitan pendientes de $45^{\circ}$ para desencadenarse, mientras que para aludes de placa y fusión 
sería suficiente con pendientes de $30^{\circ}$ y $25^{\circ}$, respectivamente (FERNÁNDEZ CAÑADAS, 2014).

Los factores intensificadores no son necesarios para el desencadenamiento de un alud, pero aumentan las probabilidades de que se produzca. Se han considerado como tales las orientaciones favorables a la aparición de placas de viento, es decir, a sotavento de los vientos dominantes, la insolación máxima y mínima recibida por las laderas entre los meses de noviembre a abril y, finalmente, las zonas de máxima permanencia de la nieve (Muñoz et al., 1995), considerándose como enclaves de mayor acumulación.

Una vez cartografiados los factores necesarios e intensificadores, se ha procedido a su análisis por medio del software de libre distribución Q GIS Dufour 2.0. Para ello se ha seguido el método boleano, reclasificando la malla ráster y otorgando valores de 1 a los píxeles que presentaran un factor necesario $o$ intensificador y 0 a los que no mostraran ninguno de ellos. Una vez obtenidas las capas ráster de todos los factores involucrados en el desencadenamiento de los aludes, se han introducido en la calculadora ráster, aplicándoles el algoritmo (factor necesario x (1+intensificadores)) (TABLA 2).

Tabla 2. Algoritmos introducidos en el SIG para la elaboración de la cartografía de susceptibilidad.

\begin{tabular}{ll}
\hline \multicolumn{1}{c}{ Tipo } & \multicolumn{1}{c}{ Algoritmo } \\
\hline Placa & Pendiente $>30^{\circ} \times(1+$ Insolación baja + Orientación a sotavento $)$ \\
Fusión & Pendiente $>25^{\circ} \times(1+$ Permanencia de la nieve + Insolación alta $)$ \\
Nievereciente & Pendiente $>45^{\circ} \times(1+$ Permanencia de la nieve + Insolación baja $)$ \\
General & Avalancha de placa + Aval. de fusión + Aval. de nieve reciente \\
\hline
\end{tabular}

FuENTE: Adaptado de FERNÁNDEZ CAÑADAS, 2014.

Los grados de susceptibilidad se han clasificado en función de la existencia o no de los factores desencadenantes, ya sean necesarios o intensificadores. De esta manera, las áreas en las que aparecían superpuestos los tres factores considerados en el algoritmo (TABLA 2) se han considerado como de susceptibilidad muy alta. Siendo áreas de susceptibilidad alta las que presentan dos de los factores superpuestos y, finalmente, zonas de susceptibilidad moderada los que solo muestran un factor desencadenante.

Los datos espaciales digitales utilizados pertenecen a la serie topográfica MTN25 (escala 1:25000) del Instituto Geográfico Nacional (Hoja 577Bohoyo), las ortofotos (tamaño de píxel $0.25 \mathrm{~m}$ ó $0.50 \mathrm{~m}$ ) obtenidas por el Plan Nacional del Ortofotografía Aérea a partir del año 2004 y los modelos 
digitales de terreno obtenido mediante sensores LIDAR (densidad media 0.5 puntos $/ \mathrm{m}^{2}$ ).

\section{III.3. Estimación estadística del máximo alcance de aludes por me- dio del modelo de regresión $\alpha-\beta$}

En lugares donde no existe un registro histórico ni otros indicadores físicos (morfológicos, vegetación o geológicos) de ocurrencia de aludes, que pudieran suministrar información sobre las características del fenómeno, la aplicación de modelos para estimar la distancia máxima de alcance resulta de gran utilidad a la hora de planificar los usos territoriales y las actividades lúdicodeportivas, ya que a través de un registro de observaciones es posible evaluar la extensión espacial de los mismos y, por tanto, ofrecer una información suficiente para elaborar estudios de peligrosidad y, en última instancia, de riesgo.

El modelo estadístico de regresión $\alpha$ - $\beta$ (LIED y BAKKEHØI, 1980; BAKKEHØIET al., 1983) fue desarrollado por el Instituto Geotécnico Noruego y aplicado con éxito en numerosas cordilleras del mundo, entre ellas España (FURDADA y VILAPLANA, 1998). Este método se basa en la correlación existente entre diversos parámetros topográficos para obtener una estimación del máximo alcance longitudinal de un alud, teniendo como referencia su trazado central (DELPARTE et al., 2008). El modelo de regresión $\alpha-\beta$ tiene las siguientes limitaciones: no ofrece información sobre la velocidad máxima del alud, la masa de nieve movilizada, la fuerza o el alcance lateral del mismo. Sin embargo, en el caso concreto del CdG, el modelo $\alpha-\beta$ resulta de gran utilidad, ya que si asumimos que un alud siempre generará un daño personal, independientemente de su magnitud, el alcance espacial del mismoserá el factor de mayor importancia para la evaluación de la peligrosidad. De esta manera, en el CdG, donde no existen infraestructuras más allá del Refugio Elola, no es necesaria la aplicación de modelos numéricos queestimen los parámetros físicos del fenómeno, lo que justifica la utilización del modelo de regresión $\alpha-\beta$ en el CdG, a pesar de sus limitaciones.

Los parámetros topográficos necesarios se han obtenido por medio de la observación directa en el terreno y el análisis de datos espaciales digitales (MDE).

- Alpha $(\alpha)$ : Se trata de la pendiente media del recorrido de la avalancha, desde su zona de salida hasta la máxima distancia observada, donde $\alpha=$ $\arctan \left(\mathrm{H}_{\alpha} / \mathrm{L}_{\alpha}\right)$.

- Beta $(\beta)$ : Corresponde a la pendiente media del perfil de la avalancha desde su punto superior hasta el punto en el que el recorrido del alud alcanza una pendiente de $10^{\circ}$. El parámetro $\beta$ se consideró inicialmente 
como el punto en que las avalanchas comenzaban a perder energía y, por tanto, a aminorar su velocidad (LIED y BAKKEHØI, 1980; BAKKEHØIET al., 1983). Sin embargo, JONES y JAMIESON (2004) descubrieron que en pendientes de longitud reducida considerar el valor de $\beta$ como $24^{\circ}$ proveía resultados más precisos. En este trabajo se ha utilizado $\beta_{24}$ para calcular el máximo alcance de un alud, siendo $\beta_{24}=$ $\arctan \left(\mathrm{H}_{\beta 24} / \mathrm{L}_{\beta 24}\right)$.

- Theta $(\Theta)$ : Corresponde a la pendiente de la zona de partida del alud en los 100 metros superiores del recorrido de la avalancha, donde $\Theta=$ $\arctan \left(100 / \mathrm{L}_{\Theta}\right)$.

- Curvatura (y"): Representa el perfil topográfico del recorrido de la avalancha considerándolo como una función polinómica de $2^{\circ}$ orden. Sin embargo, FURDADA y VILAPLANA (1998) encontraron que una función polinómica de $4^{\circ}$ orden se ajustaba mejor al perfil de la avalancha en su porción terminal, sobre todo si esta alcanzaba el fondo de valle. Una vez obtenida la recta se extrae el coeficiente a y se multiplica por dos, siendo y" $=2 \mathrm{a}$.

- Desplazamiento vertical (H): Es el desplazamiento vertical total medido a partir de la función polinómica de $2^{\circ}$ ó $4^{\circ}$ orden. Corresponde con la diferencia entre la parte superior e inferior del perfil de la avalancha.

Figura 3. Parámetros contemplados en la aplicación del modelo $\alpha-\beta$ de LIED y BAKKEHØI (1980). En este trabajo solo se tendrá en cuenta el parámetro $\beta$.

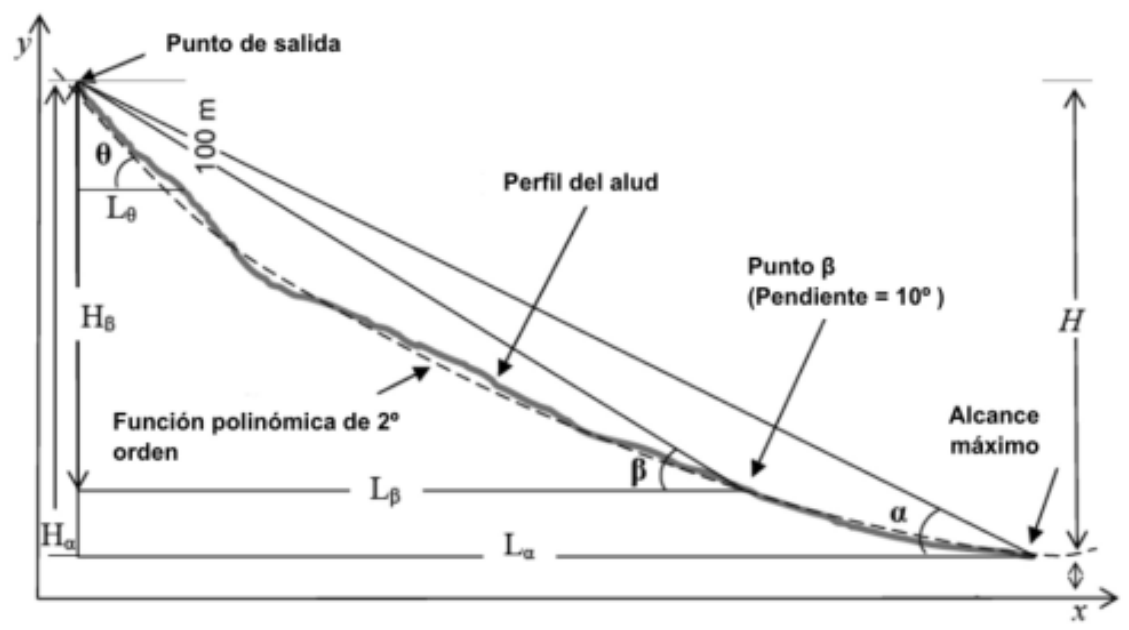

FUENTE: Elaboración propia 
Trabajos previos (MCCLUNG y LIED, 1987; FURDADA y VILAPLANA, 1998; DELPARTE et al., 2008) comprobaron que el parámetro $\beta$ es el de mayor relevancia a la hora de calcular el máximo alcance de un alud. Por esta razón, en los últimos años se ha trabajado con una versión simplificada (2) de la ecuación original (1), que solo relaciona los parámetros $\alpha-\beta$, a diferencia de la propuesta por LIED y BAKKEHØI (1980).

$$
\text { Original: } \alpha^{\prime}=b_{1} \beta+b_{2} \mathrm{H}+b_{3} \mathrm{Hy} " \Theta+b_{0} \text { (Constante) }
$$

$$
\text { Simplificada: } \alpha^{\prime}=b_{1} \beta+b_{0}
$$

Una vez obtenido el valor de $\alpha^{\prime}$, asumiendo zonas de alcance máximo horizontales, $\Delta \mathrm{L}$ será igual a:

$$
\Delta \mathrm{L}=\mathrm{L}-\mathrm{L}^{\prime}=\mathrm{H}(1 / \tan \alpha-1 / \tan (\alpha+\Delta \alpha)
$$

Para este trabajo se han utilizado 17 trayectorias observadas (FIGURA 2) y estimadas gracias a la cartografía confeccionada, de las que se han extraído los parámetros $\alpha$ y $\beta$ mediante el empleo de SIG. Posteriormente, los datos se volcaron en una hoja de cálculo donde se procedió al análisis estadístico para obtener una ecuación simplificada (2) que permita estimar el alcance máximo de los aludes en el CdG.

Una vez obtenidos los alcances máximos, se ha procedido a calcular el error porcentual de las estimaciones. La intención de esta última etapa es calibrar el modelo para que ofrezca los resultados más precisos posibles $\mathrm{y}$, de esta manera, validar su utilización en el CdG. Para llevar a cabo este cometido, se han comparado los alcances máximos $(\mathrm{m})$ observados con los estimados mediante la aplicación del modelo $\alpha-\beta$.

\section{RESULTADOS Y DISCUSIÓN}

\section{IV.1. Características nivometeorológicas del CdG}

La nieve del Macizo del Alto Gredos corresponde al tipo estacional alpino de montaña continental (STURM et al., 1995). La nieve del CdG evoluciona a gran velocidad durante la temporada, dando lugar a un manto nivoso húmedo (3-8\% de agua), con un tamaño y morfología de grano muy regular, donde abundan los tamaños gruesos (1-2 $\mathrm{mm})$ y morfologías redondeadas, con alguna aparición puntual de granos facetados y estratos de hielo. Se trata de un manto nivoso con una resistencia que oscila de media a dura.

En las vertientes orientadas hacia el norte y el este (FIGURA 4) se encuentran los neveros con mayor permanencia. Los perfiles nivológicos muestran ten- 
dencia hacia la estabilidad, la resistencia de sus capas aumenta con la profundidad. No obstante, existen ciertos factores que favorecen la ocurrencia de aludes. Por un lado, existen algunas discontinuidades en la resistencia de las mismas y los estratos de hielo son comunes. Por otro lado, la abundancia de granos gruesos $(2 \mathrm{~mm})$ y morfología redondeada de los mismos, sugieren un grado intermedio de resistencia al fallo. La humedad de la columna también se relaciona con la resistencia, por lo general, a mayor contenido de agua libre esta será menor. Sin embargo, el hecho de que la temperatura de la nieve sea cercana $\mathrm{a}-1^{\circ} \mathrm{C}$ sugiere un grado de humedad bajo, factor determinante en la generación de aludes de placa, pues el contenido en agua determina la propagación de las fracturas a través del manto nivoso (MCCLUNG y SCHAERER, 1996).

Figura 4. Perfil estratigráfico y de resistencias del manto nivoso en orientaciones norte y este en el mes de abril.
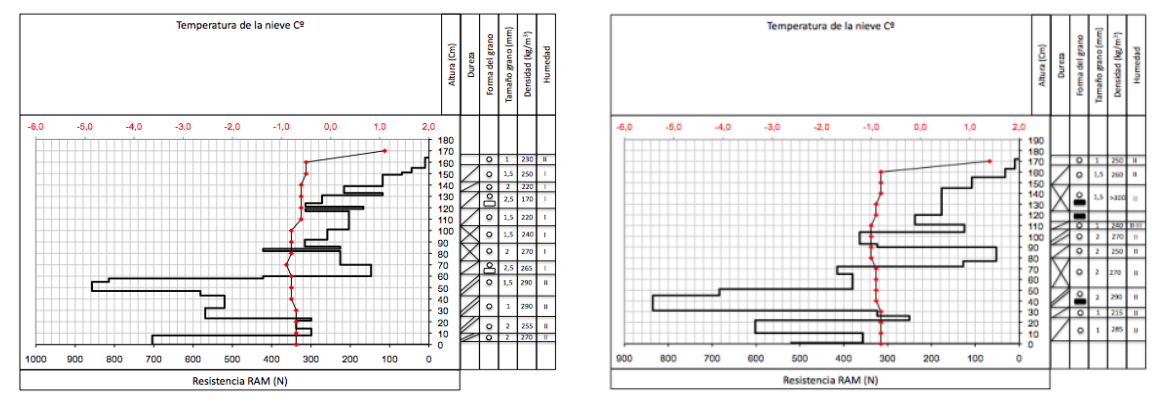

FUENTE: Elaboración propia

Figura 5. Perfil estratigráfico y de resistencias del manto nivoso en orientaciones sur y oeste en el mes de abril.
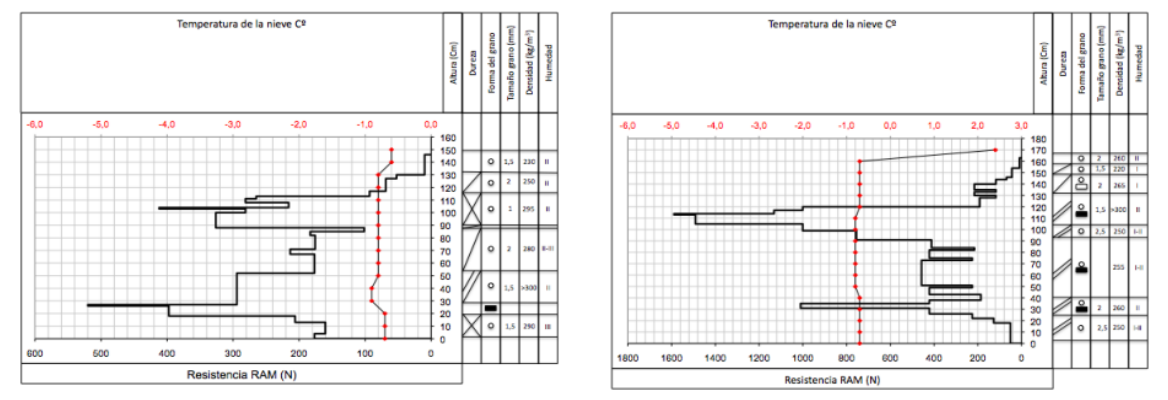

FUENTE: Elaboración propia

En las vertientes sur y oeste (FIGURA 5) donde la insolación es mayor, los espesores del manto nivoso son considerablemente menores. La característica 
principal de la nieve de estas vertientes es su acusada inestabilidad, vinculada a la heterogeneidad vertical de las resistencias de los estratos de nieve, abundando las capas de hielo de gran espesor, lo que sugiere una elevada tasa de fusión en la sección superior del manto nivoso. Esta situación favorece la probabilidad de aludes de fusión, pues las capas de hielo funcionan como estratos impermeables que acumulan agua líquida, aumentando la densidad de la nieve, disminuyendo su resistencia y dificultando la propagación de las fracturas a través del manto nivoso, imposibilitando el despegue de grandes bloques compactos.

\section{IV.2 Cartografía de susceptibilidad de aludes del CdG}

Figura 6. Mapa de susceptibilidad de aludes de placa del Circo de Gredos. Rojo: Susceptibilidad muy alta. Naranja: Susceptibilidad alta. Verde: Susceptibilidad moderada.

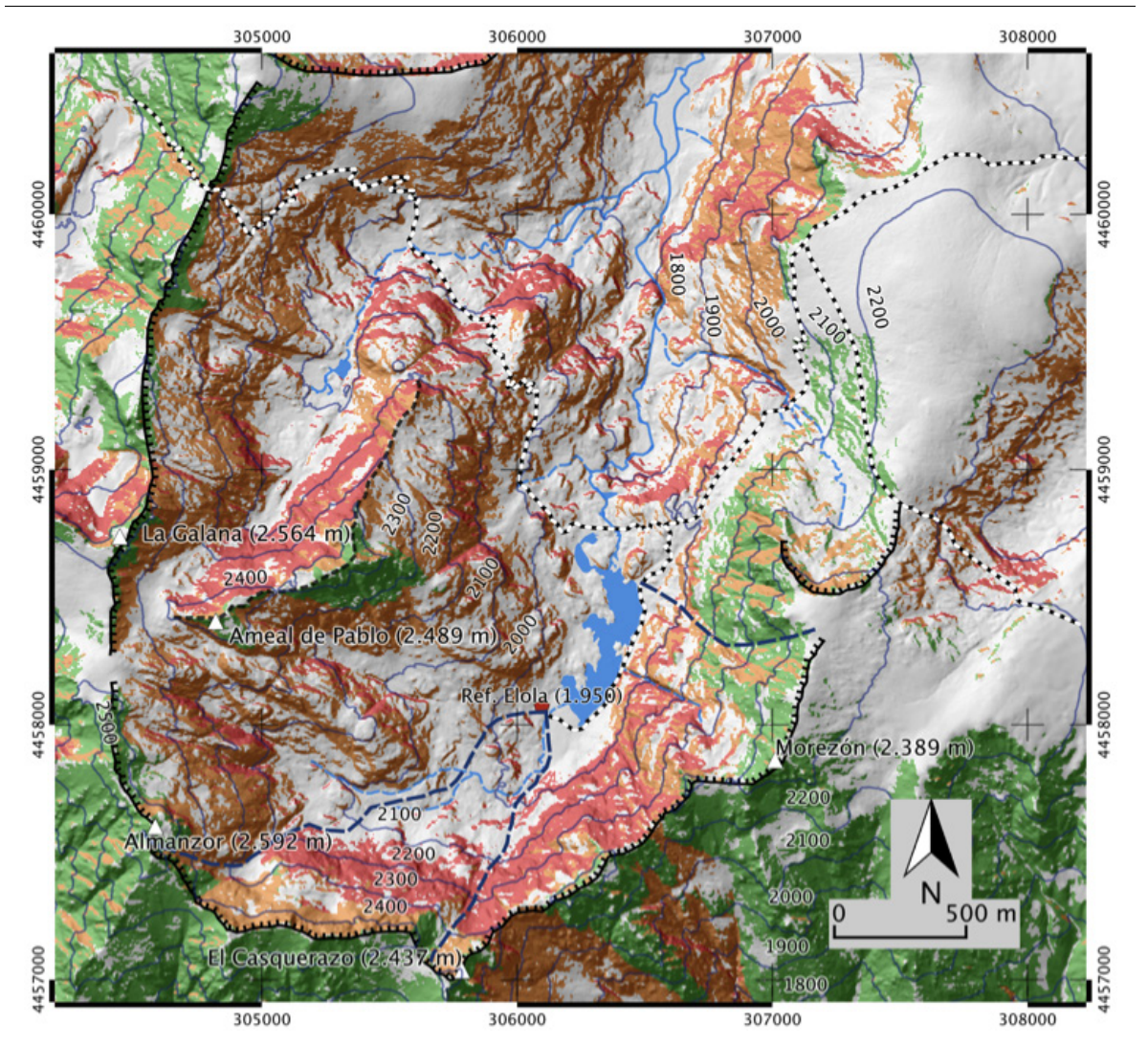

FUENTE: Elaboración propia 
En el CdG las vertientes con orientaciones N-NO son las que presentan una susceptiblidad muy alta (FIGURA 6). En estas zonas el espesor de nieve es mayor gracias al transporte eólico, lo que a su vez facilita la formación de placas de viento, las cuales pueden funcionar como capas de despegue del manto nivoso debido a su densidad y cohesión. Circunstancia favorecida por una escasa insolación solar.

Figura 7. Mapa de susceptibilidad de aludes de fusión del Circo de Gredos. Rojo: Susceptibilidad muy alta. Naranja: Susceptibilidad alta. Verde: Susceptibilidad moderada

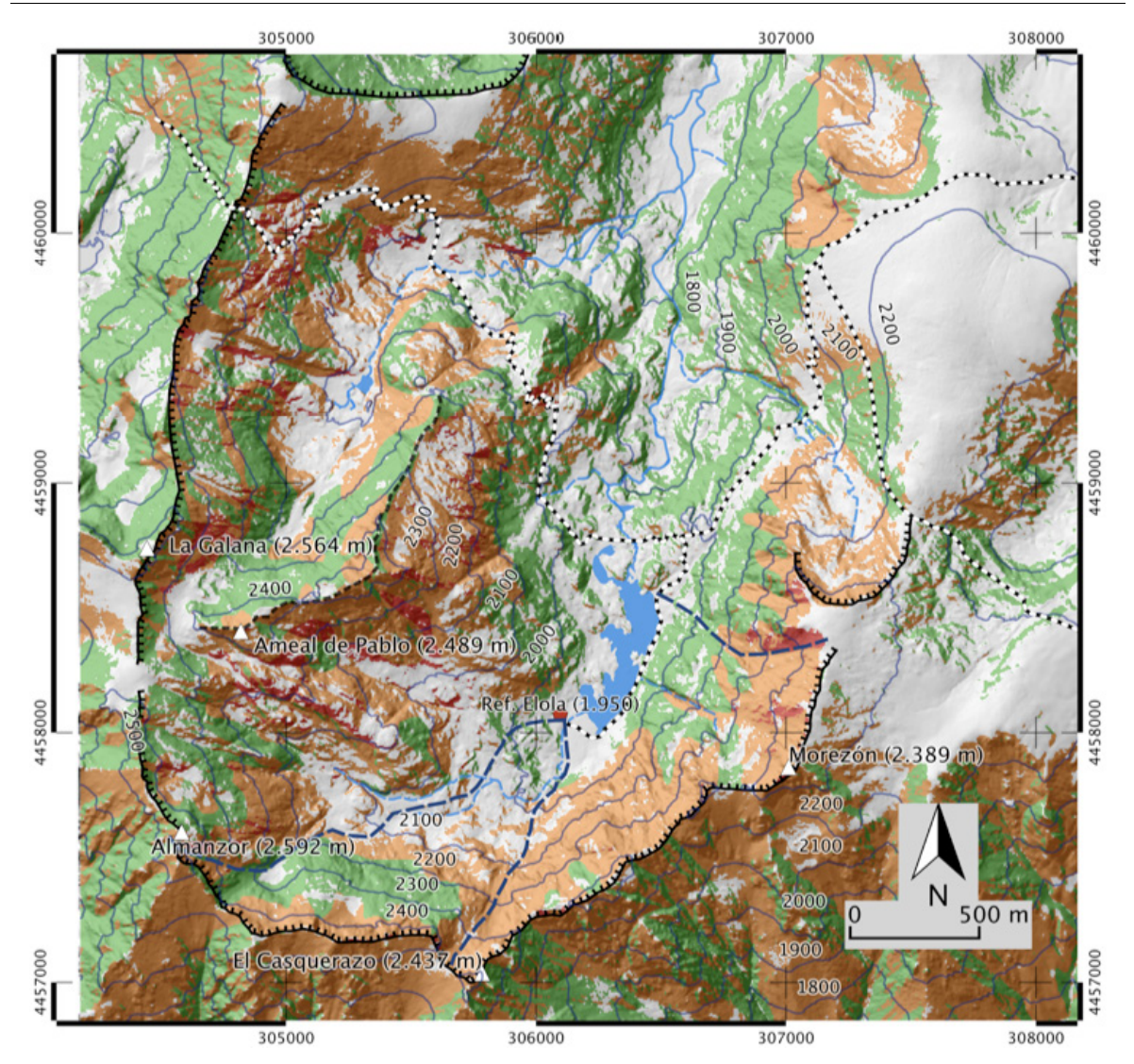

FUENTE: Elaboración propia

Los aludes de fusión son muy comunes en el CdG a finales de la temporada invernal y comienzos de la primavera, pues para que se produzca este fenómeno es necesario que el manto nivoso contenga un cierto porcentaje de agua líquida. Las laderas de orientación oeste, sur y este, es decir, las que reciben 
una mayor insolación son las más susceptibles de experimentar aludes de fusión (FIGURA 7).

\section{IV.3. Resultados de la estimación estadística del máximo alcance de aludes por medio del modelo $\alpha-\beta$}

Tras el análisis estadístico se obtuvo un modelo de regresión lineal simple utilizando los parámetros $\alpha$ y $\beta$ de los 17 recorridos de aludes observados y potenciales (FIGURA 2). De esta manera se definió una ecuación calibrada para el Circo de Gredos (4), válida para estimar el máximo alcance de los aludes.

$$
\alpha^{\prime}=0,9422 \beta+0,4608 \quad \mathrm{R}^{2}=0,94 \quad \sigma=0,645^{\circ}
$$

La ecuación extraída del modelo $\alpha-\beta$ para el CdG, parece estar en consonancia con los resultados obtenidos en otras cordilleras del mundo (TABLA 3). La diferencia más significativa se corresponde con un elevado coeficiente de correlación $\mathrm{R}^{2}(0,94)$. No obstante, trabajos previos sugieren que esta situación es normal en modelos basados únicamente en el parámetro $\beta$ (Delparte et al., 2008). Por otro lado, la desviación estándar $\left(0.645^{\circ}\right)$, obtenida de la media de $\alpha$, también resulta más elevada. Lo que significa que los alcances máximos observados son mayores que la media de los mismos, por lo que las estimaciones obtenidas podrían ser ligeramente menores que los alcances reales (LIED \&TOPPE., 1989).

Tabla 3. Comparación de los resultados obtenidos tras la aplicación del modelo $\alpha$ Ben diferentes cordilleras del mundo. Resultados de este estudio destacados en negrita.

\begin{tabular}{lrrrrrrr}
\hline \multicolumn{1}{c}{ País } & Ecuación & $R^{2}$ & $\begin{array}{r}\text { S.D. } \\
\left({ }^{\circ}\right)\end{array}$ & $N$ & $\begin{array}{r}\text { Media } \\
\alpha\left(^{\circ}\right)\end{array}$ & $\begin{array}{r}\text { Media } \\
\left.\beta()^{\circ}\right)\end{array}$ & $\begin{array}{r}\text { Media } \\
H(m)\end{array}$ \\
\hline Noruega & $\alpha^{\prime}=0,93 \beta$ & 0,86 & 2,1 & 192 & - & - & - \\
Noruega Occid. & $\alpha^{\prime}=0,90 \beta$ & 0,87 & - & 127 & 29,4 & 32,6 & 827 \\
Austria & $\alpha^{\prime}=0,94 \beta-0,83$ & 0,92 & 1,5 & 80 & - & - & - \\
Islandia & $\alpha^{\prime}=0,85 \beta$ & 0,52 & 2,2 & 44 & - & - & - \\
Columbia Mts. & $\alpha^{\prime}=0,93 \beta$ & 0,89 & 1,1 & 35 & 28,5 & 30,6 & 946 \\
Rocosas/Purcells & $\alpha^{\prime}=0,93 \beta$ & 0,75 & 1,75 & 126 & 27,8 & 29,8 & 869 \\
Cord. cost., EEUU & $\alpha^{\prime}=0,90 \beta$ & 0,74 & 1,70 & 31 & 26,8 & 29,5 & 903 \\
Alaska & $\alpha^{\prime}=0,86 \beta$ & 0,58 & - & 52 & 25,4 & 29,6 & 765 \\
Colorado & $\alpha^{\prime}=0,80 \beta$ & 0,50 & - & 130 & 22,6 & 27,4 & 641 \\
Sierra Nevada & $\alpha^{\prime}=0,76 \beta$ & 0,60 & - & 90 & 20,7 & 26,1 & 590 \\
Pirineos catalanes & $\alpha^{\prime}=0,86 \beta+1,05$ & 0,75 & 1,98 & 64 & 24,7 & 27,3 & 753 \\
\hline Circo de Gredos & $\alpha^{\prime}=0,94 \beta+0,46$ & 0,94 & 0,64 & 17 & 30,1 & 31,2 & 240 \\
\hline
\end{tabular}

FUENTE: Modificado a partir de DELPARTE et al., (2008). 
El error en las estimaciones realizadas en trayectorias de aludes conocidas para validar el modeloestá relacionado con el perfil de la trayectoria del alud. De esta manera, los corredores que se caracterizan por presentar una transición abrupta con el fondo de valle ofrecen resultados con un error aproximado del $0,83 \%$, mientras que para las trayectorias parabólicas el error se sitúa en torno al 2,08\%. Resultados coherentes con los propuestos por (FURDADA y VILAPLANA, 1998).

Figura 8. Ejemplo de aplicación del modelo $\alpha-\beta$ en el Zócalo del Almanzor, la Portilla Bermeja, Portilla de los Machos y Canal de la Mina.Amarillo: Línea central de la trayectoria; Naranja: Recorrido observado o estimado; Rojo: Alcance máximo calculado a través del modelo $\alpha-\beta$.

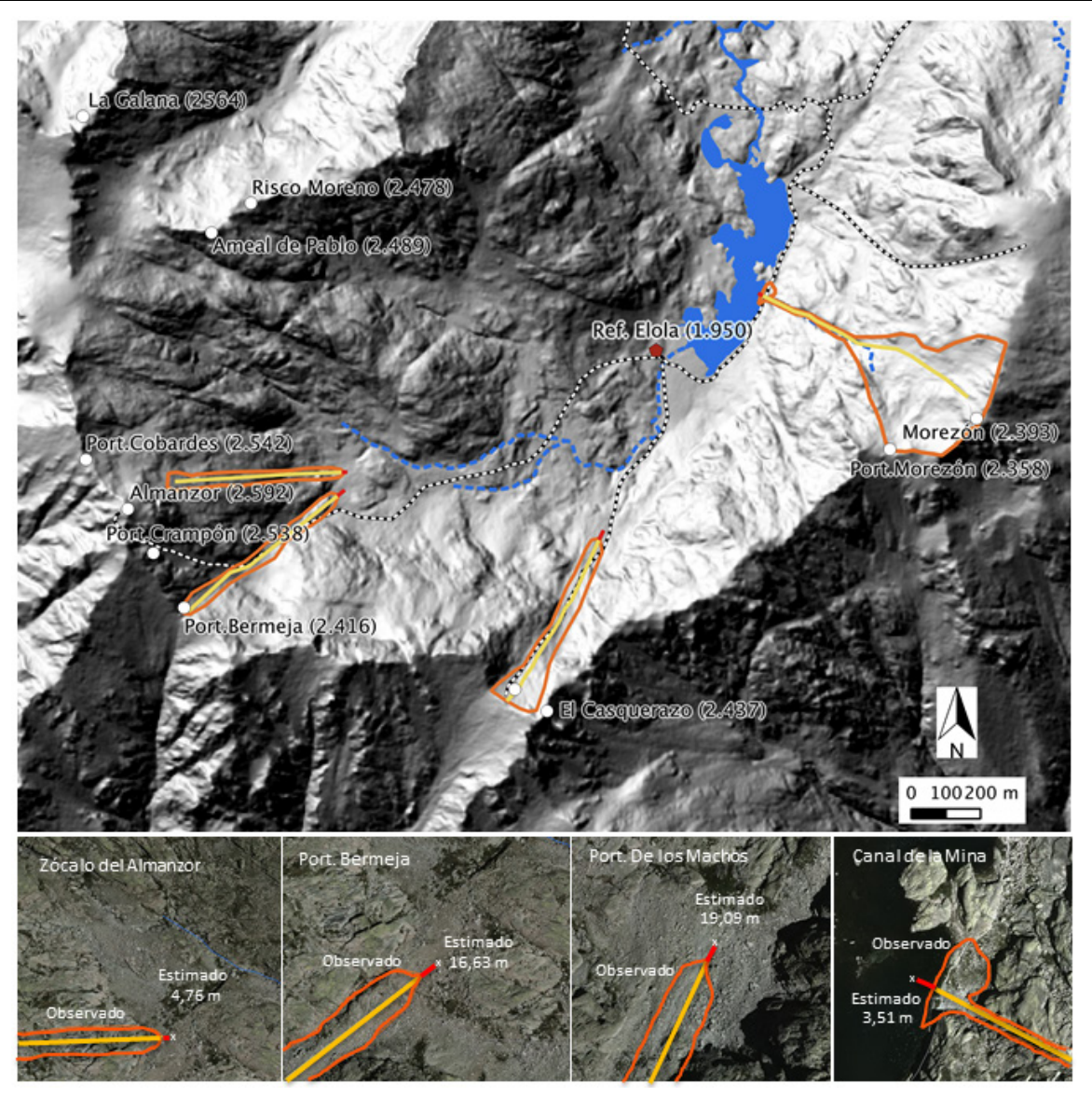

FUENTE: Elaboración propia 
A pesar de lo anterior, el error derivado de la aplicación del modelo $\alpha-\beta$ es suficientemente reducido como para justificar el empleo del mismo en el Circo de Gredos. Resultado que se ajusta a las observaciones de FURDADA y VILAPANA (1998), en el Pirineo Catalán, y JONES y JAMIESON (2004), en Canadá, donde el modelo ofrece resultados precisos para aludes medianos en pendientes de corto recorrido, los cuales presentan una elevada similitud morfológica con los ocurridos en el CdG.

Tabla 4. Parámetros topográficos y estimaciones del máximo alcance de los aludes en trayectorias de aludes conocidas.

\begin{tabular}{lrrrr}
\hline & $\begin{array}{r}\text { Port. } \\
\text { Bermeja }\end{array}$ & $\begin{array}{r}\text { Port. } \\
\text { Machos }\end{array}$ & $\begin{array}{r}\text { Canal de la } \\
\text { Mina }\end{array}$ & $\begin{array}{r}\text { Zócalo } \\
\text { Almanzor }\end{array}$ \\
\hline LongitudL $(\mathrm{m})$ & 540,18 & 532,11 & 554,01 & 478,70 \\
\hline Altura $H(\mathrm{~m})$ & 263,04 & 306,39 & 325,05 & 297,57 \\
\hline$\beta_{24}\left({ }^{\circ}\right)$ & 26,2 & 30,29 & 31,5 & 32,9 \\
\hline Morfología de la ladera & Parabólica & Parabólica & Trans. abrupta & Trans. abrupta \\
\hline Alcance estimado $(\mathrm{m})$ & $+16,63$ & $+19,09$ & $+3,51$ & $+4,76$ \\
\hline Error $(\%)$ & 3,07 & 3,58 & 0,63 & 0,99 \\
\hline
\end{tabular}

FUENTE: Elaboración propia

\section{IV.3.a. Posibles fuentes de error del modelo $\alpha-\beta$}

Existen varias fuentes potenciales de error. La más importante está relacionada con la extracción del parámetro $\beta_{24}$. Aunque existe cierta técnica para estimar la porción de la trayectoria del alud donde se encuentre el mismo (LIED y TOPPE, 1989), esta no suele ser aplicable a todos los perfiles (FURDADA y VilaPLANA, 1998). De esta manera, al no existir un único punto $\beta_{24}$, debido a la presencia de irregularidades topográficas, es necesario seleccionarlo basándose solamente en la experiencia del investigador, lo que introduce cierto grado de subjetividad.

Otra posible fuente error depende de la veracidad, precisión y lapso temporal del catálogo histórico de aludes de una zona concreta. Comúnmente, el empleo de modelos para estimar el alcance máximo de un alud se realiza en lugares donde no existe un registro sistemático de los eventos. Poseer una gran cantidad de observaciones sobre la distancia máxima alcanzada por los aludes es de vital importancia para obtener una ecuación bien calibrada, cuyos resultados se ajusten a la realidad de la zona de estudio. Por esta razón, la falta de la misma, o su poca precisión, podría derivar en la obtención de resultados con un error tan elevado que desaconsejara el empleo del modelo. 
Finalmente, hay que considerar el error vinculado con la resolución de los datos espaciales digitales. Debido a que el modelo $\alpha-\beta$ está basado en la extracción de parámetros topográficos, la resolución y precisión de los modelos digitales de elevación utilizados podría hacer variar el resultado de manera significativa (DELPARTE et al., 2008).

\section{CONCLUSIONES}

La Sierra de Gredos constituye uno de los espacios naturales con mayor ocurrencia de aludes del Sistema Central Ibérico, ya que aparecen ampliamente representados todos los factores condicionantes del fenómeno considerados en este estudio. Además, en los últimos años, ha experimentado un aumento significativo de visitantes durante la temporada invernal.

Las características del manto nivoso en el CdG varían notablemente según su localización. El manto nivoso localizado en orientaciones norte y este presenta un mayor espesor medio y un tiempo de permanencia más extenso. Las razones que explican esta situación están relacionadas con su posición a sotavento de los vientos dominantes y a la baja insolación recibida durante el invierno. Estas orientaciones tienden a presentar una estabilidad significativa al final de la temporada. Sin embargo, siguen apareciendo capas de débil cohesión y resistencia, las cuales están vinculadas con el desencadenamiento de aludes de placa.

Por otro lado, la nieve acumulada en las vertientes sur y oeste presenta un menor espesor, pues se trata de laderas orientadas a barlovento donde la nieve es movilizada por la acción eólica, y se aprecia una mayor insolación durante el periodo de permanencia de la nieve. La estructura general del manto nivoso en estas orientaciones muestra una acusada inestabilidad vertical debido a la alternancia de estratos resistentes y débiles, llamando la atención el elevado número de capas de hielo de gran espesor, cuya existencia se relaciona con los procesos de desencadenamiento de aludes de fusión.

Todas las vertientes del CdG son susceptibles de sufrir los efectos de los aludes, lo que convierte al espacio en un lugar de elevado riesgo, sobre todo para alpinistas y esquiadores, cuyas actividades se desarrollan en las laderas del circo. Por otro lado, el Refugio Elola no se encuentra en un emplazamiento peligroso.

El tipo de alud de mayor peligrosidad más frecuente espacio-temporalmente corresponde con el tipo placa. Le siguen los aludes de fusión, que cobrarían importancia en periodos de altas temperaturas invernales y al comienzo de la primavera, cuando la nieve comienza a acumular una gran cantidad de agua líquida. Finalmente, los aludes de nieve reciente serían los menos frecuentes, 
aunque cabe considerar la posibilidad de ocurrencia de aludes con características intermedias entre placa y nieve reciente.

Tras la utilización de la ecuación obtenida con el modelo de regresión $\alpha-\beta$ en aludes de alcance longitudinal conocido, se ha comprobado que esteofrece resultados precisos a la hora de estimar el máximo alcance de los aludes en el $\mathrm{CdG}$, lo que invita a su utilización a la hora de elaborar nuevas estrategias de mitigación del riesgo de aludes.

Los resultados del modelo de regresión $\alpha-\beta$ sugieren que el perfil de la trayectoria de los aludes es el parámetro de mayor importancia en la definición de la ecuación en cuestión. Por esta razón, el modelo muestra una mayor precisión en las vertientes cuya transición con el fondo de valle es abrupta. Esta situación sugiere que la elaboración de diferentes ecuaciones atendiendo a las características morfológicas de las vertientes, podría mejorar los resultados.

Finalmente, cabe reseñar la importancia de realizar este tipo de estudio en entornos de alta montaña con una elevada afluencia de visitantes durante la temporada invernal, así como la necesidad de mejorar los ya realizados mediante el seguimiento y registro continuo de las condiciones nivometeorológicas y los aludes ocurridos. De esta manera, la cartografía de susceptibilidad podría ser mejorada y ofrecer una información de mayor precisión al usuario interesado.

\section{AGRADECIEMIENTOS}

Al Parque Regional de la Sierra de Gredos por su ayuda y asistencia. Este trabajo es una contribución al proyecto CGL2013-44076-P financiado por el Ministerio de Economía y Competitividad. A Borja Robert por su inestimable ayuda durante los trabajos de campo y a los revisores de este artículo, que amablemente han contribuido a su mejora y publicación

\section{BIBLIOGRAFÍA}

ACAso, E.; Ruíz-Zapata, M. B.; PedrazA, J. y Centeno, J. (1985): «Contribución al estudio del periglaciarismo en la Sierra de Gredos». Cuaderno Lab. Xeolóxico de Laxe, 10, 115 - 132.

AEMET y IMP(2011): Atlas Climático Ibérico. AEMET e IMP, Madrid, 80 p.

BAKKEHØI, S.; DOMAAS, U.; y Lied, K. (1983): «Calculation of snow avalanche runout distance». Annals of Glaciology, 4, 24 - 29.

CArrasco, R. M.; PedraZA, J.; DomíngueZ-Villar, D.; Villa, J. y Willenbring, J. K. (2013): «The plateau glacier in the Sierra de Béjar (Iberian Central System) during its maximum extent. Reconstruction and chronology». Geomorphology, 196: 83 - 93. 
ChueCA, J. y Julián, A. (2004): «Caracterización y tipología de canales de aludes en el valle de Ordesa (Pirineo central español)». Boletín de la Real Sociedad Española de Historia Natural (Sección Geología), 99 (1-4): 93 - 103.

ChueCA, J. y Julián, A. (2010): «Cartografía de zonas probables de salida de aludes en el Alto Gállego (Pirineo Central Aragonés) mediante el empleo de Sistemas de Información Geográfica». Cuadernos de Investigación Geográfica, 36 (1), 27 41.

ChuecA, J.; Julián, A. y MontAÑéS, A. (2014): «A proposal for avalanche susceptibility mapping in the Pyrenees using GIS: the Formigal-Peyreget area (Sheet 145-I; scale 1:25.000)». Journal of Maps, 10(2), 203-210.

DelParte, D.; JAMIESON, B. y WATERS, N. (2008): «Statistical runout modeling of snow avalanches using GIS in Glacier National Park, Canada». Cold Regions Science and Technology, 54(3), 183-192.

FERNÁNDEZ CAÑADAS, J. A. (2014): Los aludes de nieve en el Macizo de Peñalara. Nota técnica 14. Agencia Estatal de Meteorología (AEMET), Madrid, 58 p.

FurdadA, G. (1996): Estudi de les allaus al Pirineu Occidental de Catalunya: predicció espacial i aplicacions de la cartografia. Geoforma Ediciones, Logroño, $315 \mathrm{p}$.

FURDADA, G. y VilAPLANA, J. M. (1998): «Statistical prediction of maximum avalanche run-out distances from topographic data in the western Catalan Pyrenees (northeast Spain)». Annals of Glaciology, 26, 285 - 288.

JONES, A. S. y JAMIESON, B. (2004): «Statistical avalanche-runout estimation for short slopes in Canada». Annals of Glaciology, 38(1), 363-372.

Julián, A. y CHUECA, J. (1999): «Cartografía de zonas probables de aludes en el valle de Ordesa (Pirineo aragonés)». Geographicalia, 37, 73 - 86.

Julián, A.; PeÑA, J. L.; ChueCA, J.; ZABAlZA, J.; LAPEÑA, A. y LÓPEZ, I. (2000): «Cartografía de zonas probables de aludes en el Pirineo aragonés: Metodología y resultados». Boletín de la AGE.;30, 119-134.

LIED, K. y BAKKEHØI, S. (1980): «Empirical calculations of snow-avalanche run-out distance based on topographic parameters». Journal of Glaciology, 26 (94), 165 177.

LIED, K. y TOPPE, R. (1989): «Calculation of maximum snow-avalanche runout distance by use of digital terrain models». Annals of Glaciology, 13, 164 - 169.

MCCLUNG, D. M. \& LIED, K. (1987): «Statistical and geometrical definition of snow avalanche runout». Cold Regions Science and Technology, 13(2), 107 - 119.

MCCLUNG, D. M. \& SCHAERER, P. (1996): Manual de avalanchas. Editorial Desnivel, Madrid, $307 \mathrm{p}$.

Muñoz, J.; Palacios, D. y DE MARCos, J. (1995): "The influence of the geomorphologic heritage on present slope dynamics. The Gredos Cirque, Spain». Pirineos, 145, 35 - 63.

MuÑoz Trigo, P. (1988): Prevención y defensa contra aludes. Aplicación práctica al Pirineo aragonés. Tesis Doctoral. Escuela Técnica Superior de Ingenieros de Montes. Universidad Politécnica de Madrid, 497 p. 
Odriozola, J. M.; Peón, A.; Ugidos, J. M.; PedrazA, J. y Fernández, P. (1978): Mapa Geológico de España 1:50.000, hoja $n^{\circ} 557$ (Bohoyo). IGME, Madrid.

Palacios, D.; De Marcos, J. y VÁZQueZ-SElem, L. (2011): «Last glacial maximum and deglaciation of Sierra de Gredos, Central Iberian Peninsula». Quaternary International, 233 (1), 16 - 26.

Palacios, D.; AndréS, N.; De Marcos, J. y VÁzQuez-Selem, L. (2012): «Maximum glacial advance and deglaciation of the Pinar Valley (Sierra de Gredos, Central Spain) and its significance in the Mediterranean context». Geomorphology, 177, 51 - 61.

Palomo, M. (2007): «Los aludes en el Circo de Piedrafita (Pirineo aragonés): el evento del 8 de febrero de 1996». Boletín Glaciológico Aragonés, 8, 61 - 83.

PAlOMO, M. (2008): «Análisis espacial de las áreas favorables al desencadenamiento de aludes en el circo de Piedrafita (Huesca)». Lucas Mallada, 13, 215 - 235.

PedrazA, J.; Carrasco, R. M.; Domínguez, D. y Villa, J. (2013): «Late Pleistocene glacial evolutionary stages in the Gredos mountains (Iberian Central System)». Quaternary International, 302, 88-100.

RODÉS I MuÑOZ, P. (1999): Análisis de los accidentes por aludes de nieve en España: Una aproximación a la revisión histórica. Ergon, Barcelona, 73 p.

RoDÉs I MUÑOZ, P. (2012): «Accidentes por aludes de nieve en España durante las diez primeras temporadas del siglo XXI». Revista de l'Associació per al Coneixement de la Neu i les Allaus, 4, 19-23.

SÁez Alagón, M. T. (1993): Atlas de zonas probables de aludes en el Valle del Aragón. Oficina de Proyectos del IGME, Zaragoza.

SÁez Alagón, M. T. (1995): Atlas de zonas probables de aludes en el Valle del Gállego. Oficina de Proyectos del IGME, Zaragoza.

Sanz, G.; Rodríguez, J.; PAscual, R.; PANTOJA, L.; Fernández CAÑAdAS, J. A.; MiQUel, E. y GARCíA CABARGA, J.A. (2013): Guía para la observación nivometeorológica. Agencia Estatal de Meteorología, Madrid.

Santos González, J.; Redondo Vega, J. M.; Gómez Villar, A. y GonzÁlez GutiÉRrez, R. B. (2010): «Los aludes de nieve en el Alto Sil (Oeste de la Cordillera Cantábrica, España)». Cuadernos de Investigación Geográfica, 36, 7 26.

SARDINERO, S. (2004): «Flora y vegetación del macizo occidental de la Sierra de Gredos (Sistema Central, España)». Guineana, 10, 474 p.

Sturm, M.; Holmgren, J. y LiSTON, G.E. (1995): «A seasonal snow cover classification system for local to global applications». Journal of Climate, 8(5), 1261-1283

VADA, J. A.; Frochoso, M. y VilaplanA, J. M. (2012): «Evaluación y cartografía del riesgo de aludes en el camino PR-PNPE 21 de acceso a la Vega de Urriellu, Picos de Europa (Noroeste de España)». Cuaternario y Geomorfología, 26 (1), 29 $-47$. 